\title{
An Empirical Approach to the Bond Additivity Model in Quantitative Interpretation of Sum Frequency Generation Vibrational Spectra
}

\author{
Hui $\mathrm{Wu}^{a \dagger}$, Wen-kai Zhang ${ }^{b \dagger}$, Wei Gan ${ }^{b}$, Zhi-feng Cui ${ }^{a}$, and Hong-fei Wang ${ }^{a, b *}$ \\ a. Department of Physics, Anhui Normal University, Wuhu, Anhui Province, China 241000; \\ b. State Key Laboratory of Molecular Reaction Dynamics, \\ Institute of Chemistry, the Chinese Academy of Sciences, Beijing, China, 100080
}

(Dated: October 29, 2018)

\begin{abstract}
Knowledge of the ratios between different polarizability $\beta_{i^{\prime} j^{\prime} k^{\prime}}$ tensor elements of a chemical group in a molecule is crucial for quantitative interpretation and polarization analysis of its SFGVS spectrum at interfaces. The bond additivity model or the hyperpolarizability derivative model along with experimentally obtained Raman depolarization ratios has been widely used to obtain such tensor ratios for the $\mathrm{CH}_{3}, \mathrm{CH}_{2}$, and $\mathrm{CH}$ groups. Successfully, such treatment can quantitatively reproduce the intensity polarization dependence in SFG-VS spectra for the symmetric (ss) and asymmetric (as) stretching modes of $\mathrm{CH}_{3}$ and $\mathrm{CH}_{2}$ groups, respectively. However, the relative intensity between the ss and as modes usually does not agree with each other within this model even for some of the simplest molecular systems, such as the air/methanol interface. This fact certainly has cast uncertainties on the effectiveness and conclusions based on the bond additivity model. One of such examples is that the as mode of $\mathrm{CH}_{3}$ group has never been observed in SFGVS spectra from the air/methanol interface, while this as mode is usually very strong for SFG-VS spectra from the air/ethanol, other short chain alcohol, as well as long chain surfactants, interfaces. In order to answer these questions, an empirical approach from known Raman and IR spectra is used to make corrections to the bond additivity model. With the corrected ratios between the $\beta_{i^{\prime} j^{\prime} k^{\prime}}$ tensor elements of the ss and as modes, all features in the SFG-VS spectra of the air/methanol and air/ethanol interface can be quantitatively interpreted within the bond additivity model. This empirical approach not only provides new understandings of the effectiveness and limitations of the bond additivity model, but also provides a practical roadmap for its application in SFG-VS studies of molecular interfaces.
\end{abstract}

\section{INTRODUCTION}

Since the first report of Sum Frequency generation Vibrational Spectroscopy (SFG-VS) experiment in early $1987, \frac{1}{\underline{1}}$ SFG-VS has been widely used to investigate various molecular interfaces, including vapor/liquid, liquid/liquid, air(vacuum gas)/solid, and liquid/solid interfaces, because SFG-VS, as one of the second order nonlinear optical processes, is interface selective and is sensitive to submonolayer changes at the molecular interface 2 3.4.5.6.7.8.9.10 Since the very beginning, quantitative interpretation of the SFG-VS spectra has been used to derive molecular orientational structure and order of the molecular interfaces under study 11.12

The key for such quantitative interpretation lies on the ability to obtain or estimate the ratios between different microscopic polarizability $\beta_{i^{\prime} j^{\prime} k^{\prime}}$ tensor elements of a chemical group in a molecule ${ }^{10,13,14}$ So far, almost all of the efforts have focused on the stretching vibrational modes. The approaches employed to obtain the $\beta_{i^{\prime} j^{\prime} k^{\prime}}$ tensor ratios are all based on the following general relationship between the second order hyperpolarizability tensors $\left(\beta_{i^{\prime} j^{\prime} k^{\prime}}^{q}\right)$ for a particular vibrational mode $q$ to the Raman polarizability derivative tensors $\left(\partial \alpha_{i^{\prime} j^{\prime}} / \partial Q_{q}\right.$, usually denote as $\left.\alpha_{i^{\prime} j^{\prime}}^{\prime}\right)$ and dipole moment derivative tensors $\left(\partial \mu_{k^{\prime}} / \partial Q_{q}\right.$, usually denote as $\left.\mu_{k^{\prime}}^{\prime}\right)$ of the $q$ th

\footnotetext{
$\dagger$ These two authors are with equal contribution.

*Authors to whom correspondence should be addressed. Email: hongfei@mrdlab.icas.ac.cn, Tel: 86-10-62555347, Fax: 8610-62563167.
}

mode, i.e. 10.13 .15

$$
\beta_{i^{\prime} j^{\prime} k^{\prime}}^{q}=-\frac{1}{2 \epsilon_{0} \omega_{q}} \frac{\partial \alpha_{i^{\prime} j^{\prime}}}{\partial Q_{q}} \frac{\partial \mu_{k^{\prime}}}{\partial Q_{q}}
$$

Here $\left(i^{\prime} j^{\prime} k^{\prime}\right)$ represents the molecular coordinates system, $\omega_{q}$ and $Q_{q}$ are the vibrational frequency, and the normal coordinates of the $q$ th vibrational mode of the molecule, respectively. Therefore, according to Eq if the proper ratios between different $\alpha_{i^{\prime} j^{\prime}}^{\prime}$ terms and the ratios between different $\mu_{k^{\prime}}^{\prime}$ terms are known, the ratios of $\beta_{i^{\prime} j^{\prime} k^{\prime}}^{q}$ elements can be readily obtained.

Among the approaches to obtain $\beta_{i^{\prime} j^{\prime} k^{\prime}}^{q}$ tensor ratios, which shall be compared in detail in Section II, the bond additivity model is based on symmetry analysis along with the Raman bond polarizability derivative theory and the bond moment theory.10.16.17.18 In some cases, it has been proven successful in quantitative interpretation of the symmetric stretching (ss) vibrational mode in SFG-VS studies 10.11 .12 .16 .17 .18 .19 .20 .21 .22 .23 .24 .25 .26 However, it often failed to quantitatively predict the SFG spectral intensity relationship between the symmetric stretching (ss) and asymmetric stretching (as) vibrational modes, as shall be discussed in detail in Section II. Therefore, the limitations and effectiveness of the bond additivity model still needs to be investigated.

In this report, we shall employ an complete empirical approach to the bond additivity model by using experimental IR and Raman spectra to correct the discrepancies between the $\alpha_{i^{\prime} j^{\prime}}^{\prime}$ and $\mu_{k^{\prime}}^{\prime}$ tensors of the molecular groups. The effectiveness of this approach shall be demonstrated with the ss and as modes of the $\mathrm{CH}_{3}$ group in $\mathrm{CH}_{3} \mathrm{OH}$ and $\mathrm{CH}_{3} \mathrm{CD}_{2} \mathrm{OH}$ molecules. We shall show 
that with the experimentally corrected $\alpha_{i^{\prime} j^{\prime}}^{\prime}$ and $\mu_{k^{\prime}}^{\prime}$ tensor relationships, the $\beta_{i^{\prime} j^{\prime} k^{\prime}}$ tensor ratios calculated from Eq1 can be used successfully to quantitatively interpret detailed polarization dependence in SFG-VS spectra of the vapor $/ \mathrm{CH}_{3} \mathrm{OH}$ and vapor $/ \mathrm{CH}_{3} \mathrm{CD}_{2} \mathrm{OH}$ interfaces. This empirical approach overcomes the limitations of the current simple bond additivity model, and it also provides a practical roadmap for its application in SFGVS studies of molecular interfaces. This approach also demonstrates how to use molecular IR and Raman spectra in the bulk liquid or gaseous phases for quantitative understanding of the SFG-VS vibrational spectra of the same molecule at the interface, and vise versa.

\section{BACKGROUND}

Here we briefly compare the different approaches for obtaining $\beta_{i^{\prime} j^{\prime} k^{\prime}}^{q}$ tensor ratios, and discuss how and why the bond additivity model failed to predict the SFG spectral intensity relationship between the symmetric stretching (ss) and asymmetric stretching (as) vibrational modes

\section{A. Three approaches for the $\beta_{i^{\prime} j^{\prime} k^{\prime}}^{q}$ tensor ratios}

As a 3 rd rank tensor, $\beta_{i^{\prime} j^{\prime} k^{\prime}}^{q}$ has $3 \times 3 \times 3=27$ tensor elements in total. In the most ideal case, the ratios between all of these tensor elements should be determined apriori. Recently, Hore et al. proposed a whole molecule approach based on a general scheme for ab initio calculation of the vibrational hyperpolarizability of any IR- and Raman-active mode, regardless of the molecular symmetry or complexity of the structure, and application of this approach to the $-\mathrm{OSO}_{3}$ headgroup of a surfactant molecule at the air/water interface was also demonstrated 14 With the tremendous advancement of the capability and availability of the ab initio computation methods, the advantage of this whole molecule approach is apparent. However, because this approach is purely computational, its general accuracy can be affected by the limitations of the current ab initio computational methods in reproducing the Raman and IR spectra intensities, as well as in dealing with the problem of mode couplings, such as Fermi resonances. Nevertheless, this attempt does provide an promising alternative solution to the limitations of other existing approaches.

Unlike the whole molecule approach mentioned above, these other approaches all employed the local mode assumption of the particular molecular group. The bond additivity model even further employed the local mode assumption of the particular bonds. With the knowledge of the symmetry of the molecular groups, the number of non-zero $\beta_{i^{\prime} j^{\prime} k^{\prime}}^{q}$ tensor elements can be significantly reduced; accordingly, the number of non-zero $\alpha_{i^{\prime} j^{\prime}}^{\prime}$ elements of the symmetry group can also be significantly reduced ${ }^{15,27}$ For example, the $\mathrm{CH}_{3}$ and $\mathrm{CH}_{2}$ groups can be treated with $C_{3 v}$ and $C_{2 v}$ symmetries, respectively. Thus, their non-zero $\beta_{i^{\prime} j^{\prime} k^{\prime}}^{q}$ elements for the stretching vibrational modes are reduced to 11 and 7 , respectively, ${ }^{15,27}$ There have been discussions on whether the local mode treatment of the $\mathrm{CH}_{3}$ and $\mathrm{CH}_{2}$ groups with $C_{3 v}$ and $C_{2 v}$ symmetries, respectively, is valid 28.29 However, such concerns have not been a serious issue because it has been generally accepted in molecular spectroscopy textbook that '...in the case of $\mathrm{C}-\mathrm{H}$ stretches, the high frequency of the local vibration of the $\mathrm{C}-\mathrm{H}$ bond tends to uncouple that motion from that of the rest of the molecule' ${ }^{30}$ In addition, slight deviation from $C_{3 v}$ symmetry of the $\mathrm{CH}_{3}$ group in actual molecules can be treated with small perturbations, as discussed by Hirose et $a l^{16}$.

One such approach used experimental Raman depolarization ratio to determine the $\beta_{i^{\prime} j^{\prime} k^{\prime}}^{q}$ ratios of the symmetric stretching (ss) mode of $C_{3 v}$ and the stretching mode of $C_{\infty v}$ groups, because both groups have only two independent $\beta_{i^{\prime} j^{\prime} k^{\prime}}^{q}$ terms for the ss mode under the local group mode assumption, i.e., there is only one $\beta_{i^{\prime} j^{\prime} k^{\prime}}^{q}$ ratio $R=\beta_{a a c} / \beta_{c c c}=\beta_{b b c} / \beta_{c c c}$ need to be determined, ${ }^{10.31}$ The coordinates $(a, b, c)$ is define as in Fig 1. This direct Raman polarization ratio method worked because according to Eq1 $R=\alpha_{a a}^{\prime} / \alpha_{c c}^{\prime}=\alpha_{b b}^{\prime} / \alpha_{c c}^{\prime}$ for the ss mode of $C_{3 v}$ and the stretching mode of $C_{\infty v}$ groups, and it can be obtained from Raman experimental depolarization measurement 10 This approach has been widely used and worked successfully because it is purely empirical, and it solely depends on the reliability of the particular Raman depolarization measurement. 10,31.32,33,34

One may surmise that even though the group itself may not strictly observe the $C_{3 v}$ or $C_{\infty v}$ symmetry, because this $R$ value is the effective value obtained from the $\mathrm{Ra}$ man spectral features with the assumption of $C_{3 v}$ or $C_{\infty v}$ symmetry, it should dutifully reproduce back to the same features of the Raman spectra with the same assumption of symmetry. Because this $R$ value is strictly the same for the corresponding $\beta_{i^{\prime} j^{\prime} k^{\prime}}^{q}$ ratio according to Eq. 1] it is not hard to see that with this $R$ value and the same assumption of symmetry, the related features in the SFGVS spectral can be reproduced. This is why this approach has worked so well for the ss mode of the $C_{3 v}$ and the stretching mode of the $C_{\infty v}$ symmetry groups 10.31 .32 .33

However, this approach can not be applied to $C_{2 v}$ group, which has three instead of two independent $\beta_{i^{\prime} j^{\prime} k^{\prime}}^{q}$ terms for its ss mode $\frac{10}{10}$ Furthermore, this approach does not deal with the ratio between the $\beta_{i^{\prime} j^{\prime} k^{\prime}}^{q}$ of symmetric and asymmetric stretching modes of the $C_{3 v}$ molecular group. Therefore, it can not be used to address the relative SFG intensity of the ss and as modes of the $C_{3 v}$ molecular group.

An useful alternative method in the SFG-VS literature is the widely used bond additivity model, which can give $\beta_{i^{\prime} j^{\prime} k^{\prime}}^{q}$ ratios of the $C_{3 v}, C_{2 v}, C_{\infty v}$ and other symmetry groups, including both of their ss and as modes $10,11,12,16,17,18,19,20,21,22,23,24,25,26$ The bond additivity model can also be called the bond polarizability derivative model. As Hore et al. pointed out recently, 14 the key to the bond additivity model is that through symmetry analysis the polarizability tensors of the individual bond stretches $\left(C_{\infty v}\right)$ are coupled to produce the normal mode coordinate of a particular molecular group 


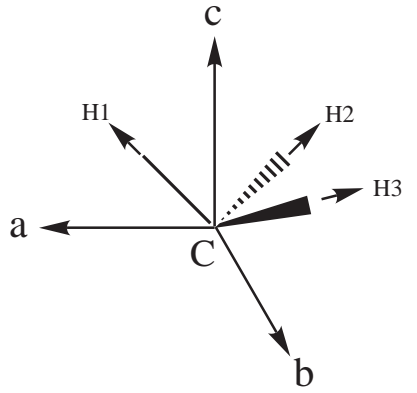

FIG. 1: Molecule-fixed(abc) axis for $C_{3 v}$ symmetry. Illustrated with the $\mathrm{CH}_{3}$ group.

$\left(C_{3 v}, C_{2 v}\right.$, etc. $)$ 16.17 There are two ways to determine the polarizability tensors ratios of the individual bond stretches, i.e. $r=\beta_{\xi \xi \zeta} / \beta_{\zeta \zeta \zeta}=\beta_{\eta \eta \zeta} / \beta_{\zeta \zeta \zeta}$, where $(\xi \eta \zeta)$ is the single bond fixed coordinates with $\zeta$ as the primary axis of the single bond. One way is through theoretical ( $a b$ initio) calculation of the Raman tensors of the single bond,, 35 and the other way is through experimentally measured Raman depolarization ratio, or Raman intensity ratio between the ss and as modes, $, 10,16,17$ on the basis that the single bond posses $C_{\infty v}$ symmetry and that $r=\alpha_{\xi \xi}^{\prime} / \alpha_{\zeta \zeta}^{\prime}=\alpha_{\eta \eta}^{\prime} / \alpha_{\zeta \zeta}^{\prime}$ for a single bond according to Eq1

The detailed formula and the effectiveness, as well as some limitations, of the bond polarizability model was critically reviewed recently 10 So far, this model have been used for study of $\mathrm{H}_{2} \mathrm{O}$ molecule at the air/water interfaces, ${ }_{19.20 .21 .22}-\mathrm{CH}_{2}$ - groups ${ }^{18.24 .25 .26}$ and occasionally $-\mathrm{CH}_{3}$ groups ${ }^{11,12,17,23}$ at various molecular interfaces. The unique success of the bond additivity model has been on $\mathrm{H}_{2} \mathrm{O}$ molecules and $-\mathrm{CH}_{2}$ - groups to which direct Raman polarization ratio method can not be applied as mentioned above. In terms of $-\mathrm{CH}_{3}$ or other $\mathrm{C}_{3 v}$ groups, the bond additivity model is essentially the same thing as the direct raman depolarization ratio method for the ss mode. Another unique advantage of the bond additivity model is that it can give the $\beta_{i^{\prime} j^{\prime} k^{\prime}}^{q}$ ratio between the ss and as modes for the $\mathrm{C}_{3 v}$ group ${ }^{10,26}$ However, some intrinsic weakness of the bond additivity model has limited its effectiveness on interpretation of the relative intensity of the ss and as modes in actual SFG-VS spectra. This is discussed in detail as in the followings.

The bond additivity model uses the Raman bond polarizability derivative tensors $\alpha_{i^{\prime} j^{\prime}}^{\prime}$ and the bond moment derivative tensors $\mu_{k^{\prime}}^{\prime}$ to calculate $\beta_{i^{\prime} j^{\prime} k^{\prime}}$ tensors according to Eq1 10,17 In Raman and IR spectroscopy studies, the bond polarizability theory and the bond moment theory have been used to interpret the IR and Raman spectral intensity ${ }^{36}$ As we have known, even though the Raman bond polarizability derivative model have worked well with the intensities of the ss modes, it has not been very effective on the relative intensity between the ss and as modes of the same molecular group 36.37 .38 .39 .40 Furthermore, the simple bond moment hypothesis, also called the zero-order bond moment theory, as used in the bond additivity model in SFG-VS, has not been successful in interpretation of IR intensities ${ }^{36.41 .42 .43 .44}$ This is because the simple bond moment theory essentially neglects all coupling effect between the single bonds even within the same molecular group. Therefore, modified bond moment theory which includes such and other coupling effects has been proposed,, 36 but such modified theory becomes complicated by introduced many unknown parameters in order to address the coupling terms. In short, these facts certainly limit the effectiveness of the bond additivity model for quantitative interpretation of the SFG-VS spectra.

However, with all these problems, two of the important and successful features of the bond additivity model should not be overlooked. Firstly, the bond additivity model rooted deeply into the concept of molecular symmetry analysis, which is the basis for the polarization selection rules and has provided clear physical picture for understanding of the polarization and orientational dependence in SFG-VS, ${ }^{10.25 .26}$ as well as all other spectroscopic techniques. ${ }^{45}$ Secondly, the direct Raman polarization ratio method has been quantitatively successful in many of the SFG-VS applications ${ }^{10.31 .32 .33 .34}$ Since a full computational solution for quantitative interpretation of the SFG-VS spectra is yet not within our reach as mentioned above, it can certainly be beneficial to extend the bond additivity model if we can use the core concept for its successes, and try to directly address the limitations in the consisting Raman and IR theories.

\section{B. Failure of the bond additivity model}

The failure of the bond additivity model came to our attention in our recent study of SFG-VS spectra of vapor/alcohol interfaces of $\mathrm{C}_{1}-\mathrm{C}_{8}$ alcohols in the $\mathrm{CH}$ stretching vibration region between 2800 to $3000 \mathrm{~cm}^{-1} \cdot 10,26$ The fact has been long known that there has been no observation of the $\mathrm{CH}_{3}$-as peak, which should be around $2970 \mathrm{~cm}^{-1}$, in the SFG-VS spectra of the vapor/methanol interface, $12,26,46,47,48,49,50,51,52$ while for longer chain alcohols $\left(\mathrm{C}_{2}-\mathrm{C}_{8}\right.$ alcohols), the $\mathrm{CH}_{3^{-}}$ as peak is apparently very strong in their SFG-VS spectra 26.48 .52 Even though the bond additivity model can seemingly explain why the longer chain alcohols can have stronger $\mathrm{CH}_{3}$-as peak in the SFG-VS spectra of their vapor/liquid interfaces than that of the vapor/methanol interface, ${ }^{10}$ it also predict that the $\mathrm{CH}_{3}$-as mode of methanol should be observable in the sps SFGVS spectrum.

Fig 2 presents the SFG-VS spectra and the calculated polarization dependence against the $\mathrm{CH}_{3}$ orientational angle $\theta$ from the interface normal using the bond additivity model in the ssp, ppp, and sps polarizations for two different experimental configurations. The SFG-VS intensity is proportional to $\sec ^{2}(\beta) d^{2} R(\theta)$, and the detail of calculation has been presented previously. ${ }^{10,26}$ It is to be noted that calculation of the ss mode intensity uses $\beta_{c c c}^{2}$ as unit, and the as mode uses $\beta_{a c a}^{2}$ as unit. It is clear that for both experimental configura- 

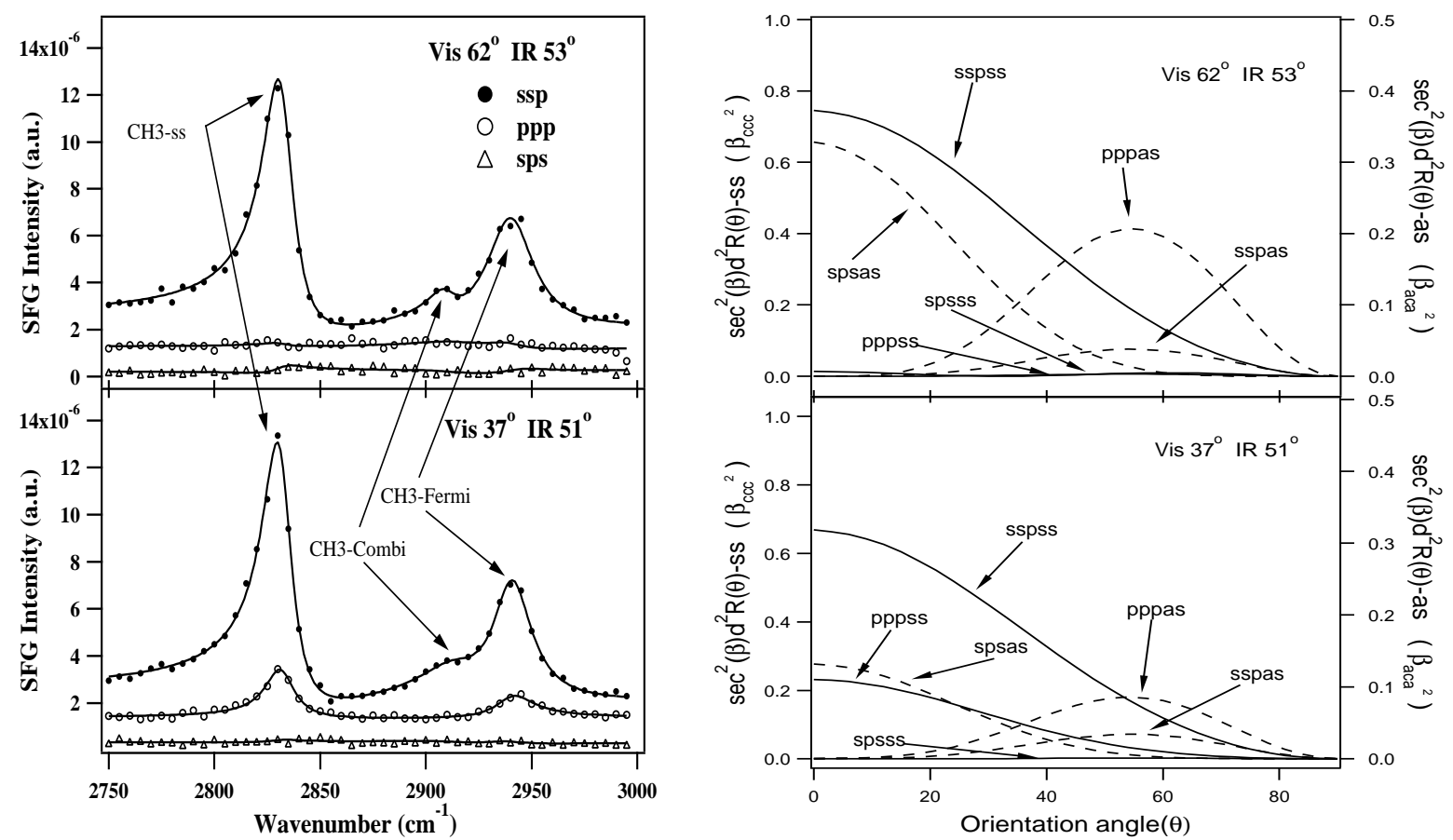

FIG. 2: SFG spectra of the air/methanol interface in two different experimental configurations with different incident angles. The $\beta$ in $\sec ^{2}(\beta)$ is the angle between the SFG signal beam and the surface normal. It is determined by the visible and IR incident angles. The SFG intensity is proportional to $\sec ^{2}(\beta) d^{2} R(\theta)$ in units of $\beta_{c c c}^{2}$ or $\beta_{a c a}^{2}$ for the ss and as modes, respectively. The solid lines in the two figure on the left are fittings with Lorentzian lineshapes.
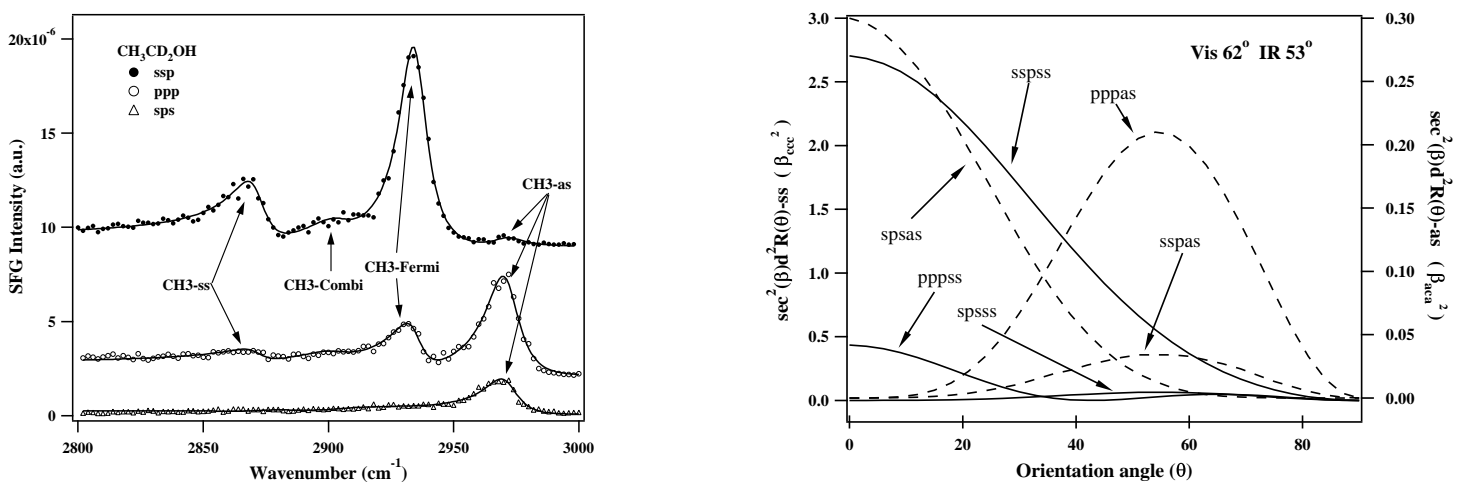

FIG. 3: SFG-VS spectra of the vapor $/ \mathrm{CH}_{3} \mathrm{CD}_{2} \mathrm{OH}$ interface in ssp, ppp and sps polarizations with incident angles of Vis $=62^{\circ}$ and $\mathrm{IR}=53^{\circ}$ in the $\mathrm{SFG}$ experiment. Because the $\mathrm{CH}_{2}$ group is deuterated, therefore, all spectral features belong to the $\mathrm{CH}_{3}$ group. The solid lines are fittings with Lorentzian lineshapes.

tions, $\mathrm{CH}_{3}$-as mode around $2970 \mathrm{~cm}^{-1}$ can not be observed in the SFG-VS spectra. This tells us that the missing of the $\mathrm{CH}_{3}$-as mode peak is not something accidental. With the bond additivity model, methanol has $\beta_{c c c} / \beta_{a c a}=1.1$ for its $\mathrm{CH}_{3}$ group, as calculated in Section III. Therefore, from the calculation results in Fig 2 $\mathrm{CH}_{3}$-as should be experimentally observable in at least one of the three polarizations in comparison to the intensities of the $\mathrm{CH}_{3}$-ss mode. Recent measurements have determined that for vapor/methanol interface the $\mathrm{CH}_{3}$ orientation is close to the interface normal. ${ }^{10,50,51.53} \mathrm{With}$ this $\mathrm{CH}_{3}$ group orientation, the bond additivity model predicted that the $\mathrm{CH}_{3}$-ss mode in the ppp polarization can be observed in the second experimental configuration $\left(\mathrm{VIS}=37^{\circ}\right.$ and $\mathrm{IR}=51^{\circ}$ ), and this spectral feature was indeed observed with the expected intensity as indi- cated in Fig 2. This fact certainly suggests the effectiveness of the bond additivity model calculations for the $\mathrm{CH}_{3}$-ss mode of methanol molecule. Accordingly, if the bond additivity calculation is also effective for the as mode, the $\mathrm{CH}_{3}$-as mode intensity in the sps polarization should be more than $1 / 3$ of that of the $\mathrm{CH}_{3}$-ss mode in the ssp polarization of the first experimental configuration. However, no such $\mathrm{CH}_{3}$-as mode has been ever observed 12.26 .46 .47 .48 .49 .50 .51 .52 This clearly demonstrates the failure of the bond additivity model in dealing with the $\mathrm{CH}_{3}$-as mode, even though the intensities of the $\mathrm{CH}_{3}$-ss and $\mathrm{CH}_{3}$-ss-Fermi modes can be quantitatively explained as in Fig[2]

In comparison, Fig 3 presents the SFG-VS spectra and the bond additivity model calculation of vapor $/ \mathrm{CH}_{3} \mathrm{CD}_{2} \mathrm{OH}$ interface with the incident angles 
$\mathrm{Vis}=62^{\circ}$ and $\mathrm{IR}=53^{\circ}$ in the SFG-VS experiment. It is clear that the $\mathrm{CH}_{3}$-as mode around $2970 \mathrm{~cm}^{-1}$ in the ppp polarization is even much bigger than the $\mathrm{CH}_{3}$-ss mode around $2870 \mathrm{~cm}^{-1}$ in the ssp polarization. This is consistent with the bond additivity model value of $\beta_{c c c} / \beta_{a c a}=0.30$ for the $\mathrm{CH}_{3}$ group of $\mathrm{CH}_{3} \mathrm{CD}_{2} \mathrm{OH}$ molecule, as calculated in Section IV. Then, why the bond additivity model works for the $\mathrm{CH}_{3}$-as mode of $\mathrm{CH}_{3} \mathrm{CD}_{2} \mathrm{OH}$ but not that of $\mathrm{CH}_{3} \mathrm{OH}$ ?

Even though the bond additivity model and the direct Raman polarization ratio method have been apparent success in quantitative interpretation of the polarization dependence and orientational analysis of the $\mathrm{CH}_{3}$ groups from the SFG-VS spectra of its $\mathrm{CH}_{3}$-ss mode, 10.50 .51 their inability and discrepancy to address the as mode intensities of the $\mathrm{CH}_{3}$ group in $\mathrm{CH}_{3} \mathrm{CD}_{2} \mathrm{OH}$ and $\mathrm{CH}_{3} \mathrm{OH}$ molecules are certainly unpleasant, and put uncertainties to these models. However, as discussed above, the bond additivity model is still the most useful model in obtaining the $\beta_{i^{\prime} j^{\prime} k^{\prime}}^{q}$ tensor ratios. We realized that the effectiveness of these models on the ss mode of the $C_{3 v}$ and the stretching mode of the $C_{\infty v}$ symmetry lies on the fact that their $R$ values are empirically obtained. On the other hand, the failures of these models also lie on the fact that those other ratios were not set on the same empirical basis. In order to address these problems and to have better understanding of the bond additivity model, we propose an complete empirical approach in order to correct the failures of the bond additivity model. This approach includes the full empirical treatment of the ss vs. as ratios of the raman and IR tensors, in addition to the successful empirical treatment of the ss mode.

\section{BOND ADDITIVITY MODEL AND ITS EMPIRICAL CORRECTION}

In a recently review on quantitative treatment on SFGVS from molecular interfaces, we discussed related issues on the bond additivity model in detail ${ }^{10}$ We pointed out that the original expression given by Hirose et al ${ }^{16.17}$ needed to be corrected for some minor errors, and the correct expressions for the $\mathrm{C}_{3 v}, \mathrm{C}_{2 v}$ and $\mathrm{C}_{\infty v}$ groups were presented $\stackrel{10}{\underline{10}}$ We also presented some good examples to demonstrate the validity of the bond additivity model for quantitative analysis of SFG-VS spectra, especially for interpretation of the ss modes of the $\mathrm{C}_{3 v}, \mathrm{C}_{2 v}$ and $\mathrm{C}_{\infty v}$ groups, and we also pointed out that further detailed examinations of this model were still needed $\underline{10}$

Therefore, here we shall try to keep the successful part of the bond additivity model, which is to use the experimental Raman depolarization ratio of the ss mode to obtain the single bond Raman polarizability derivative tensor ratio $r$, and try to derive the expressions for the Raman and IR intensity expressions for the ss and as mode of the $\mathrm{C}_{3 v}$ group, in order to obtain the corrections factors for the $\alpha_{i^{\prime} j^{\prime}}^{\prime}$ ratios and $\mu_{k^{\prime}}^{\prime}$ ratios between the ss and as modes from the experimental Raman and IR spectra, respectively. We hope this approach can produce the corrected $\beta_{i^{\prime} j^{\prime} k^{\prime}}^{q}$ tensor ratios between the ss and as modes, and can be used to quantitatively address the above discussed problems in the SFG-VS spectra of the vapor $/ \mathrm{CH}_{3} \mathrm{OH}$ and vapor $/ \mathrm{CH}_{3} \mathrm{CD}_{2} \mathrm{OH}$ interfaces.

For the stretching vibrational modes of a molecular group with $\mathrm{C}_{3 v}$ symmetry, there are a single symmetric mode $\left(A_{1}\right)$ and a doubly degenerated asymmetric mode $(E)$, with the following relationships between the $\beta_{i^{\prime} j^{\prime} k^{\prime}}, \alpha_{i^{\prime} j^{\prime}}^{\prime}$ and $\mu_{k^{\prime}}^{\prime}$ tensors when there is no electronic resonances: ${ }^{10,54.55}$

For the $A_{1}$ (symmetric) mode:

$$
\begin{aligned}
\text { SFG-VS } & \beta_{a a c}=\beta_{b b c}=R \beta_{c c c} \\
\text { Raman: } & \alpha_{a a}^{\prime}=\alpha_{b b}^{\prime}=R \alpha_{c c}^{\prime} \\
\text { IR: } & \mu_{c}^{\prime}
\end{aligned}
$$

For the E (asymmetric) mode:

$$
\begin{array}{cl}
\text { SFG-VS } & \beta_{a c a}=\beta_{b c b}=\beta_{c a a}=\beta_{c b b} \\
& \beta_{a a a}=-\beta_{b b a}=-\beta_{a b b}=-\beta_{b a b} \\
\text { Raman: } & \alpha_{a c}^{\prime}=\alpha_{c a}^{\prime}=\alpha_{b c}^{\prime}=\alpha_{c b}^{\prime} \\
& \alpha_{a a}^{\prime}=-\alpha_{b b}^{\prime}=-\alpha_{a b}^{\prime}=-\alpha_{b a}^{\prime} \\
\text { IR: } & \mu_{a}^{\prime}=\mu_{b}^{\prime}
\end{array}
$$

It is to be noted that the $\alpha_{a a}^{\prime}$ and $\alpha_{b b}^{\prime}$ tensors of the symmetric mode are different from the $\alpha_{a a}^{\prime}$ and $\alpha_{b b}^{\prime}$ tensors of the asymmetric mode.

For a rotationally isotropic interface, the four asymmetric $\beta_{i^{\prime} j^{\prime} k^{\prime}}$ terms, namely $\beta_{a a a}=-\beta_{b b a}=-\beta_{a b b}=$ $-\beta_{b a b}$, are dumb tensors, which would not appear in the non-zero elements of the $3 \mathrm{rd}$ rank macroscopic susceptibility $\chi_{i j k}$ tensors 10 Therefore, if $R=\beta_{a a c} / \beta_{c c c}$ is known from the direct Raman depolarization ratio method, in addition, only the ratio $\beta_{c c c} / \beta_{a c a}$ need to be obtained for all useful $\beta_{i^{\prime} j^{\prime} k^{\prime}}$ tensor ratios $\frac{10.17}{17}$ According to Eq[1, one has,

$$
\frac{\beta_{c c c}}{\beta_{a c a}}=\frac{\omega_{a s y m}}{\omega_{s y m}} \frac{\alpha_{c c}^{\prime}}{\alpha_{a c}^{\prime}} \frac{\mu_{c}^{\prime}}{\mu_{a}^{\prime}}
$$

Therefore, with the frequencies of the symmetric and asymmetric modes ( $\omega_{\text {sym }}$ and $\omega_{\text {asym }}$, respectively) known, only the Raman tensor ratio $\alpha_{c c}^{\prime} / \alpha_{a c}^{\prime}$ and the IR tensor ratio $\mu_{c}^{\prime} / \mu_{a}^{\prime}$ need to be known to make the calculation of $\beta_{c c c} / \beta_{a c a}$.

The IR tensor ratio $\mu_{c}^{\prime} / \mu_{a}^{\prime}$ can be directly obtained from the IR intensity ratio of the symmetric and asymmetric modes. However, in the simple bond additivity model, the $\mu_{c}^{\prime} / \mu_{a}^{\prime}$ ratio was not experimentally corrected 10.17

$$
\frac{I_{s y m}^{I R}}{I_{a s y m}^{I R}}=\left(\frac{\mu_{c}^{\prime}}{\mu_{a}^{\prime}+\mu_{b}^{\prime}}\right)^{2}=\left(\frac{\mu_{c}^{\prime}}{2 \mu_{a}^{\prime}}\right)^{2}
$$

In order to get $R$ and $\alpha_{c c}^{\prime} / \alpha_{a c}^{\prime}$, the following Raman polarizability theory need to be invoked.

When vertically linear polarized light is used for the Raman experiment, the following relationships for the Raman depolarization ratio and Raman intensity ratio in the parallel polarization to the individual Raman polarizability derivative tensors $\left(\alpha_{i^{\prime} j^{\prime}}^{\prime}\right)$ are generally valid for all molecular groups ${ }^{54.56 .57}$ 


$$
\begin{aligned}
\rho & =\frac{3 \gamma^{2}}{45 \alpha^{2}+4 \gamma^{2}} \\
\frac{I_{\text {sym }}^{\text {Raman }}}{I_{\text {asym }}^{\text {Raman }}} & =\frac{\alpha_{\text {sym }}^{2}+\frac{4}{45} \gamma_{\text {sym }}^{2}}{\alpha_{\text {asym }}^{2}+\frac{4}{45} \gamma_{\text {asym }}^{2}}
\end{aligned}
$$

with the definition of

$$
\begin{aligned}
\alpha= & \frac{1}{3}\left(\alpha_{a a}^{\prime}+\alpha_{b b}^{\prime}+\alpha_{c c}^{\prime}\right) \\
\gamma^{2}= & \frac{1}{2}\left[\left(\alpha_{a a}^{\prime}-\alpha_{b b}^{\prime}\right)^{2}+\left(\alpha_{b b}^{\prime}-\alpha_{c c}^{\prime}\right)^{2}\right. \\
& \left.+\left(\alpha_{c c}^{\prime}-\alpha_{a a}^{\prime}\right)^{2}+6\left({\alpha_{a b}^{\prime}}^{2}+\alpha_{b c}^{\prime 2}+\alpha_{c a}^{\prime}{ }^{2}\right)\right]
\end{aligned}
$$

The molecular coordinates are defined as in Fig प If natural light is used for the Raman spectra measurement, the factor 4/45 used in Eq11] should be 7/45, and the depolarization ratio becomes $\rho=6 \gamma^{2} /\left(45 \alpha^{2}+7 \gamma^{2}\right) ! \underline{54.56 .57}$

In this case, if one puts Eq 3 into Eq[10] because there are only three non-zero symmetric tensors, a unique $R$ value (generally $1 \leq R \leq 4$ for $\mathrm{C}_{3 v}$ groups, and $0 \leq R \leq 1$ for $\mathrm{C}_{\infty v}$ groups) can be obtained from the symmetric tensors through the experimental value of the Raman depolarization ratio $\rho$ of the $C_{3 v}$ symmetric mode, 10.13

$$
\begin{aligned}
\rho_{\text {sym }} & =\frac{3 \gamma_{\text {sym }}^{2}}{45 \alpha_{\text {sym }}^{2}+4 \gamma_{\text {sym }}^{2}} \\
& =\frac{3}{4+5[(1+2 R) /(R-1)]^{2}}
\end{aligned}
$$

Here the Eq14 for obtaining $R$ is the so called direct Raman depolarization ratio method because $R$ directly satisfies Eq 2 according to Eq ${ }^{10,31}$

However, since the raman depolarization ratio for the asymmetric mode $\rho_{\text {asym }}=3 / 4$ is always a constant, no knowledge of the asymmetric tensors can be learned from the direct Raman depolarization ratio method. Therefore, one now have to employ the bond additivity model to make such connection through Eq11.

In the bond additivity model, the single bond property is used to calculate the property of the whole group. Following Hirose et al.'s formulation,,${ }^{17.58}$ the Raman polarizability derivative of the single bond can be related to the following three non-zero tensor elements with the following relationship,

$$
\frac{\partial \alpha_{\xi \xi}}{\partial \Delta \zeta}=\frac{\partial \alpha_{\eta \eta}}{\partial \Delta \zeta}=r \frac{\partial \alpha_{\zeta \zeta}}{\partial \Delta \zeta}
$$

in which $\zeta$ is the primary axis of the single bond coordinates, and the stretching vibration displacement $\Delta \zeta$ from its equilibrium position is along the single bond. Generally, for the single bond, $0 \leq r \leq 1.10$ This is to say, the single bond is treated with $C_{\infty v}$ symmetry. In the bond additivity model,$\stackrel{10,16,17,58}{ }$ we define the following,

$$
\begin{aligned}
& a_{0}=\left(\frac{\partial \alpha_{\zeta \zeta}}{\partial \Delta \zeta}\right)_{0} \\
& m_{0}=\left(\frac{\partial \mu_{\zeta}}{\partial \Delta \zeta}\right)_{0} \\
& G_{A_{1}}=\frac{1+2 \cos \tau}{M_{A}}+\frac{1}{M_{B}} \\
& G_{E}=\frac{1-\cos \tau}{M_{A}}+\frac{1}{M_{B}}
\end{aligned}
$$

in which $\tau$ is the tetrahedral angle between two single $A$ - $B$ bonds, $M_{A}$ and $M_{B}$ are the atomic masses of the atom $A$ and $B$ of the $A B_{3}$ with $\mathrm{C}_{3 v}$ symmetry. Therefore, $G_{A_{1}}$ and $G_{E}$ are the inverse reduced masses of the $A_{1}$ and $E$ normal modes, respectively, with the normal mode coordinates defined as,

$$
\begin{aligned}
& Q_{A_{1}}=\left(\Delta r_{1}+\Delta r_{2}+\Delta r_{3}\right) /\left(3 G_{A_{1}}\right)^{1 / 2} \\
& Q_{E, a}=\left(2 \Delta r_{1}-\Delta r_{2}-\Delta r_{3}\right) /\left(6 G_{E}\right)^{1 / 2} \\
& Q_{E, b}=\left(\Delta r_{2}-\Delta r_{3}\right) /\left(2 G_{E}\right)^{1 / 2}
\end{aligned}
$$

in which $\Delta r_{i}$ is the bond displacement vector along the direction of the $i$ th bond, with the $\mathrm{C}_{3 v}$ coordinates defined as in Fig प Then according to the bond additivity model, we have the following expressions for the $\alpha_{i^{\prime} j^{\prime}}^{\prime}$ and $\mu_{k^{\prime}}^{\prime}$ tensors of the $\mathrm{C}_{3 v}$ group.

For the $A_{1}$ (symmetric) mode:

$$
\begin{aligned}
\alpha_{a a}^{\prime} & =\alpha_{b b}^{\prime} \\
& =\frac{1}{2} a_{0}\left[(1+r)-(1-r) \cos ^{2} \tau\right]\left(3 G_{A_{1}}\right)^{\frac{1}{2}} \\
\alpha_{c c}^{\prime} & =a_{0}\left(\cos ^{2} \tau+r \sin ^{2} \tau\right)\left(3 G_{A_{1}}\right)^{\frac{1}{2}} \\
\mu_{c}^{\prime} & =-m_{0} \cos \tau\left(3 G_{A_{1}}\right)^{\frac{1}{2}}
\end{aligned}
$$

For the $E$ (asymmetric) mode:

$$
\begin{aligned}
\alpha_{a c}^{\prime} & =\alpha_{c a}^{\prime}=\alpha_{b c}^{\prime} \\
& =\alpha_{c b}^{\prime}=\frac{1}{2} a_{0}(r-1) \sin \tau \cos \tau\left(6 G_{E}\right)^{\frac{1}{2}} \\
\alpha_{a a}^{\prime} & =-\alpha_{b b}^{\prime}=-\alpha_{a b}^{\prime} \\
& =-\alpha_{b a}^{\prime}=\frac{1}{4} a_{0}(1-r) \sin ^{2} \tau\left(6 G_{E}\right)^{\frac{1}{2}} \\
\mu_{a}^{\prime} & =\mu_{b}^{\prime}=\frac{1}{2} m_{0} \sin \tau\left(6 G_{E}\right)^{\frac{1}{2}}
\end{aligned}
$$

It is to be noted that the above expressions is derived with the assumption that the $C_{3 v}$ group closely assumes a normal tetrahedral structure. For most the $\mathrm{CH}_{3}$ groups under our investigation, this is generally a good assumption. In order to simplify calculations, here we use $\cos \tau=-1 / 3$, which is exact for the normal tetrahedral angle $\tau \approx 109.5^{\circ}$. Therefore, with $M_{C}=12$ and $M_{H}=1$, we have $G_{A_{1}} / G_{E}=37 / 40$.

In order to make the bond additivity model work, the single bond value of $r$ need to be determined. The $r$ value can be determined from computations, $\stackrel{35}{5}$ or from Raman experimental measurement. ${ }^{10.16 .17 .58}$. With above $\alpha_{i^{\prime} j^{\prime}}^{\prime}$ expressions put into Eq[10 and Eq[1] we have the following two expressions which can each produce an $r$ value 
from the experimental Raman depolarization ratio or the Raman intensity ratio between the ss and the as modes, respectively. For the Raman measurement with a linearly polarized light, we have,

$$
\begin{gathered}
\rho_{\text {sym }}=\frac{0.75}{1+11.25[(1+2 r) /(1-r)]^{2}} \\
\frac{I_{\text {sym }}^{\text {Raman }}}{I_{\text {asym }}^{\text {Raman }}}=\frac{45(1+2 r)^{2}+4(1-r)^{2}}{32(1-r)^{2}} \frac{G_{A_{1}}}{G_{E}} \\
\quad=\frac{45(1+2 r)^{2}+4(1-r)^{2}}{8(1+8 r)^{2}}\left(\frac{\alpha_{c c}^{\prime}}{\alpha_{a c}^{\prime}}\right)^{2}
\end{gathered}
$$

with the following relationships,

$$
\begin{aligned}
& \frac{\alpha_{c c}^{\prime}}{\alpha_{a c}^{\prime}}=\frac{1+8 r}{2-2 r}\left(\frac{G_{A_{1}}}{G_{E}}\right)^{\frac{1}{2}} \\
& \frac{\mu_{c}^{\prime}}{\mu_{a}^{\prime}}=\frac{1}{2}\left(\frac{G_{A_{1}}}{G_{E}}\right)^{\frac{1}{2}} \\
& \frac{\beta_{c c c}}{\beta_{a c a}}=\frac{\omega_{\text {asym }}}{\omega_{\text {sym }}} \frac{1+8 r}{4(1-r)} \frac{G_{A_{1}}}{G_{E}}
\end{aligned}
$$

Some of the Raman spectra were measured with naturally polarized light, then the Eq17] and Eq 18 becomes,

$$
\begin{gathered}
\frac{I_{\text {sym }}^{\text {Raman }}}{I_{\text {asym }}^{\text {Raman }}}=\frac{45(1+2 r)^{2}+7(1-r)^{2}}{56(1-r)^{2}} \frac{G_{A_{1}}}{G_{E}} \\
=\frac{45(1+2 r)^{2}+7(1-r)^{2}}{14(1+8 r)^{2}}\left(\frac{\alpha_{c c}^{\prime}}{\alpha_{a c}^{\prime}}\right)^{2}
\end{gathered}
$$

One would naturally expect that Eq16 and Eq17 (or $\mathrm{Eq}$ 221) would end up with the same $r$ value from Raman experiment measurement on the same molecule ${ }^{17}$ However, experimental data have shown that it has generally not been the case. Therefore, in the bond additivity model, Eq16 and Eq17 can not be used at the same time. This fact has not been clearly recognized since the first derivation of the bond additivity model by Hirose et al ${ }^{17}$ Now the question is whether one of them can be used, or both are not good at all. As we have known that the direct Raman polarization ratio method using Eq14 involves only with the symmetric Raman tensors of the group, and it has been widely and successfully employed 10.31 .32 .33 .34 As has been shown before, the bond additivity model with the single bond $r$ value from $\mathrm{Eq}[16$ is equivalent to direct raman depolarization ratio method with $\mathrm{Eq}{ }_{14}^{10}$ Therefore, it is reasonable to stick with the $r$ value obtained from $\mathrm{Eq} 16$ and then try to experimentally correct the $\alpha_{c c}^{\prime} / \alpha_{a c}^{\prime}$ ratio with Eq 18 by using the $r$ value obtained from Eq[16] We surmise such correction directly from the Raman depolarization ratio measurement and the Raman intensity measurement can correct the value for the asymmetric tensors, and in the meantime keep the relationship between the symmetric tensors intact.

Here Eq20 from the bond additivity model can be directly tested with IR spectral measurement using Eq9 As we have know from the discussion in Section II, it would generally fail because this simple bond moment model has not included the coupling between the single bonds. Therefore, in order to get the correct $\beta_{c c c} / \beta_{a c a}$ ratio according to $\mathrm{Eq}\left[\right.$ the $\mu_{c}^{\prime} / \mu_{a}^{\prime}$ ratio also needs to be corrected with the measured IR spectra using Eq 9

We hope by putting the corrected $\alpha_{c c}^{\prime} / \alpha_{a c}^{\prime}$ and $\mu_{c}^{\prime} / \mu_{a}^{\prime}$ ratios back into $\mathrm{Eq} 8$ the corrected $\beta_{c c c} / \beta_{a c a}$ ratio thus obtained can be used to address the failures of the bond additivity model discussed in Section IIB. If we can do so, it shall put the uncertain parameters used in the bond additivity model on a solid empirical basis. In the next section we shall used the well established IR and Raman data of liquid $\mathrm{CH}_{3} \mathrm{OH}$ and $\mathrm{CH}_{3} \mathrm{CD}_{2} \mathrm{OH}$ molecules in the previous literatures to demonstrate how this empirical approach to the bond additivity model works. The terms and expressions for the $C_{2 v}$ groups are listed in the Appendix.

\section{RESULTS AND DISCUSSION}

\section{A. Bond Additivity Model Results}

The Raman depolarization ratio for the ss mode of the $\mathrm{CH}_{3}$ group in $\mathrm{CH}_{3} \mathrm{OH}$ and $\mathrm{CH}_{3} \mathrm{CH}_{2} \mathrm{OH}$ molecules in bulk liquid at the room temperature measured with polarized laser light are $\rho=0.014$ and $\rho=0.053$, respectively 56 Photoacoustic stimulated Raman measurement of $\mathrm{CH}_{3} \mathrm{CH}_{2} \mathrm{OH}, \mathrm{CH}_{3} \mathrm{CD}_{2} \mathrm{OH}$ and $\mathrm{CD}_{3} \mathrm{CH}_{2} \mathrm{OH}$ in the gas phase gave $\rho=0.060 \pm 0.015$ for all three molecules $\frac{59}{5}$ These values are in good agreement with each other. Because the previous Raman experiment claimed to have higher accuracy (error of $\rho$ was claimed to be 0.0007 ), and was measured with bulk liquids, here we adopt the value $\rho=0.053$ for $\mathrm{CH}_{3} \mathrm{CD}_{2} \mathrm{OH}$ in this work. From $\mathrm{Eq} 14$ and $\mathrm{Eq} 16$, the $R$ and $r$ values for the $\mathrm{CH}_{3}$ group in $\mathrm{CH}_{3} \mathrm{OH}$ and $\mathrm{CH}_{3} \mathrm{CH}_{2} \mathrm{OH}$ molecules can be directly calculated.

Therefore, for $\mathrm{CH}_{3} \mathrm{OH}, R=1.7$ and $r=0.28$; while for $\mathrm{CH}_{3} \mathrm{CH}_{2} \mathrm{OH}, R=3.4$, and $r=0.026$.

Using the two $r$ values, the following values can be obtained from the uncorrected bond additivity model for the $\mathrm{CH}_{3}$ group in $\mathrm{CH}_{3} \mathrm{OH}$ and $\mathrm{CH}_{3} \mathrm{CH}_{2} \mathrm{OH}$, using the three equations $\mathrm{Eq}$ [19. $\mathrm{Eq} 21$.

For $\mathrm{CH}_{3} \mathrm{OH}$, with $r=0.28, \omega_{\text {sym }}=2836 \mathrm{~cm}^{-1}$ and $\omega_{\text {asym }}=2987 \mathrm{~cm}^{-1}$, we have,

$$
\frac{\alpha_{c c}^{\prime}}{\alpha_{a c}^{\prime}}=2.2 ; \quad \frac{\mu_{c}^{\prime}}{\mu_{a}^{\prime}}=0.48 ; \quad \frac{\beta_{c c c}}{\beta_{a c a}}=1.1
$$

For $\mathrm{CH}_{3} \mathrm{CD}_{2} \mathrm{OH}$, with $r=0.026, \omega_{\text {sym }}=2870 \mathrm{~cm}^{-1}$ and $\omega_{\text {asym }}=2976 \mathrm{~cm}^{-1}$, we have,

$$
\frac{\alpha_{c c}^{\prime}}{\alpha_{a c}^{\prime}}=0.60 ; \quad \frac{\mu_{c}^{\prime}}{\mu_{a}^{\prime}}=0.48 ; \quad \frac{\beta_{c c c}}{\beta_{a c a}}=0.30
$$

These values were the same as calculated in the literature, and were used to make the calculations for Fig 2 and Fig $3^{10}$ 
TABLE I: Integrated $\operatorname{Raman}^{56}$ and $\operatorname{IR}^{60}$ band intensities of liquid methanol at $298 \mathrm{~K}$. All values are normalized to the strongest peak in the Raman and IR spectra, respectively.

\begin{tabular}{|c|c|c|c|c|}
\hline & Band & $\nu\left(\mathrm{cm}^{-1}\right)$ & $\mathrm{FWHH}\left(\mathrm{cm}^{-1}\right)$ & Intensity \\
\hline \multirow[t]{5}{*}{ Raman } & $\mathrm{CH}_{3}-\mathrm{ss}$ & 2836 & 20 & 1.00 \\
\hline & $\mathrm{CH}_{3}-\mathrm{Combi}$ & 2885 & 39 & 0.16 \\
\hline & $\mathrm{CH}_{3}-\mathrm{Combi}$ & 2918 & 28 & 0.26 \\
\hline & $\mathrm{CH}_{3}$-Fermi & 2944 & 24 & 0.84 \\
\hline & $\mathrm{CH}_{3}$-as & 2987 & 40 & 0.20 \\
\hline \multirow[t]{5}{*}{ IR } & $\mathrm{CH}_{3}$-ss & 2833 & 24 & 0.60 \\
\hline & $\mathrm{CH}_{3}$-Combi & 2872 & 39 & 0.18 \\
\hline & $\mathrm{CH}_{3}-\mathrm{Combi}$ & 2911 & 26 & 0.98 \\
\hline & $\mathrm{CH}_{3}$-Fermi & 2946 & 37 & 1.00 \\
\hline & $\mathrm{CH}_{3}$-as & 2983 & 47 & 0.70 \\
\hline
\end{tabular}

\section{B. Empirical Corrections of Bond Additivity Model Results}

In order to make empirical corrections of the $\alpha_{c c}^{\prime} / \alpha_{a a}^{\prime}$ and $\mu_{c}^{\prime} / \mu_{a}^{\prime}$ ratios in the bond additivity model, the relative intensities of the $\mathrm{CH}_{3}$-ss and $\mathrm{CH}_{3}$-as peaks in the Raman and IR spectra need to be known.

A detailed band fitting of the Raman spectra from a polarized Raman measurement of liquid $\mathrm{CH}_{3} \mathrm{OH}$ at $298 \mathrm{~K}$ were reported by Griffiths et al. in 1972,56 The integrated band intensity of the four C-H stretching bands thus obtained are listed in Table The intensity ratio between the $\mathrm{CH}_{3}$-ss and $\mathrm{CH}_{3}$-as is thus $1.00 / 0.20=5.00$, which is used in Eq18 to calculate the corresponding $\alpha_{c c}^{\prime} / \alpha_{a c}^{\prime}$.

A detailed band fitting of the IR spectra of liquid $\mathrm{CH}_{3} \mathrm{OH}$ at $298 \mathrm{~K}$ were reported by Bertie et al. in $1997 \stackrel{60}{\underline{6}}$ The integrated band intensity of the C-H stretching bands are also listed in Table It is to be noted that the Raman and IR band positions and band widths (Table (I) are in very good agreement with each other. The intensity ratio between the $\mathrm{CH}_{3}$-ss and $\mathrm{CH}_{3}$-as is thus $0.60 / 0.70=0.86$, which is used in $\mathrm{Eq} 9$ to calculate the corresponding $\mu_{c}^{\prime} / \mu_{a}^{\prime}$ value.

The Raman and IR spectra of 12 kind of deuterated ethanol molecule were systematically studies by Perchard and Josien in 1968 61 Fig 4 shows the Raman (measured with polarized light) and IR spectra of pure liquid $\mathrm{CH}_{3} \mathrm{CD}_{2} \mathrm{OH}$ at $300 \mathrm{~K}$ reproduced from Perchard and Josien's paper. With the reproduced spectra, we performed band fitting and listed the integrated band intensities in Table III therefore, the intensity ratios between the $\mathrm{CH}_{3}$-ss and $\mathrm{CH}_{3}$-as of the Raman and IR spectra are $0.36 / 0.67=0.54$, and $0.20 / 1.00=0.20$, respectively. Comparing with the values for $\mathrm{CH}_{3} \mathrm{OH}$ above, it is clear that the relative $\mathrm{CH}_{3}$-as band Raman and IR intensities are many times stronger for $\mathrm{CH}_{3} \mathrm{CD}_{2} \mathrm{OH}$ than those for $\mathrm{CH}_{3} \mathrm{OH}$.

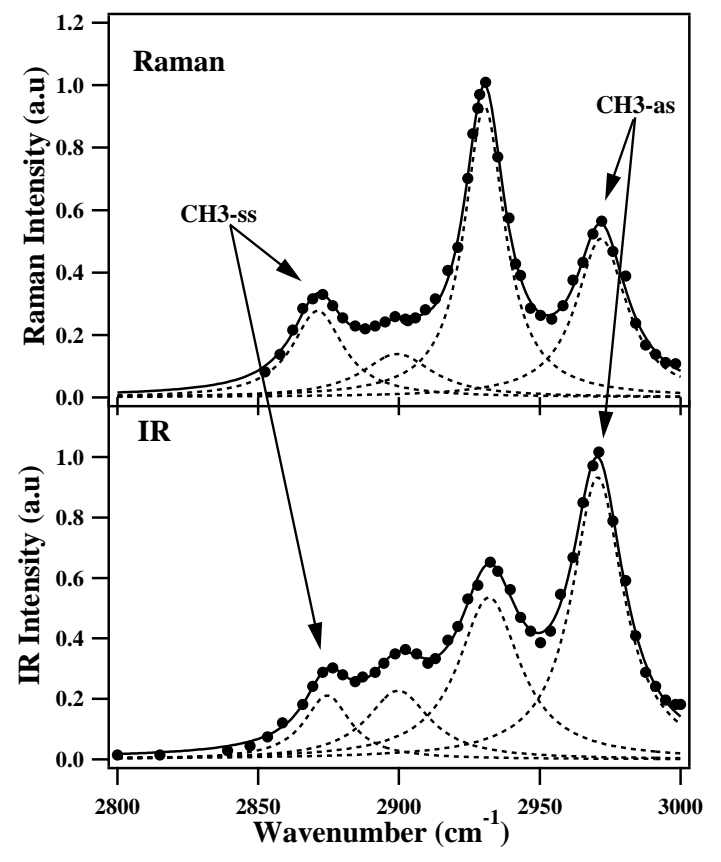

FIG. 4: Raman and IR spectra (solid dots, normalized to the strongest peak in each spectra) of pure liquid $\mathrm{CH}_{3} \mathrm{CD}_{2} \mathrm{OH}$ at $300 \mathrm{~K}$, redrawn from previous literature ${ }^{61}$ The dashed lines are fittings with Gaussian lineshape. The base lines are determined by the best Gaussian group fittings. The area of each Gaussian curve is integrated and normalized as the corresponding peak intensity listed in Table

TABLE II: Integrated Raman and IR band intensities of liquid $\mathrm{CH}_{3} \mathrm{CD}_{2} \mathrm{OH}$ at $300 \mathrm{~K}$. All values are normalized to the strongest peak in the Raman and IR spectra, respectively.

\begin{tabular}{llccc}
\hline \multicolumn{2}{c}{ Band } & $\nu\left(\mathrm{cm}^{-1}\right)$ & FWHH & Intensity \\
\hline Raman & $\mathrm{CH}_{3}$-ss & 2871 & 22 & 0.36 \\
& $\mathrm{CH}_{3}$-Combi & 2899 & 30 & 0.25 \\
& $\mathrm{CH}_{3}$-Fermi & 2930 & 17 & 1.00 \\
& $\mathrm{CH}_{3}$-as & 2972 & 22 & 0.67 \\
\hline $\mathrm{IR}$ & $\mathrm{CH}_{3}$-ss & 2875 & 20 & 0.20 \\
& $\mathrm{CH}_{3}$-Combi & 2900 & 27 & 0.29 \\
& $\mathrm{CH}_{3}$-Fermi & 2932 & 27 & 0.69 \\
& $\mathrm{CH}_{3}$-as & 2971 & 22 & 1.00 \\
\hline
\end{tabular}

Now the empirical correction for the bond additivity model can be readily made with $\mathrm{Eq} 9 \mathrm{Eq} 21$ and $\mathrm{Eq} 8$ as the following.

For $\mathrm{CH}_{3} \mathrm{OH}$, with $r=0.28, \omega_{\text {sym }}=2836 \mathrm{~cm}^{-1}$ and $\omega_{\text {asym }}=2987 \mathrm{~cm}^{-1}$, we have,

$$
\frac{\alpha_{c c}^{\prime}}{\alpha_{a c}^{\prime}}=1.9 ; \quad \frac{\mu_{c}^{\prime}}{\mu_{a}^{\prime}}=1.9 ; \quad \frac{\beta_{c c c}}{\beta_{a c a}}=3.8
$$

For $\mathrm{CH}_{3} \mathrm{CD}_{2} \mathrm{OH}$, with $r=0.026, \omega_{\text {sym }}=2871 \mathrm{~cm}^{-1}$ and $\omega_{\text {asym }}=2972 \mathrm{~cm}^{-1}$, we have,

$$
\frac{\alpha_{c c}^{\prime}}{\alpha_{a c}^{\prime}}=0.34 ; \quad \frac{\mu_{c}^{\prime}}{\mu_{a}^{\prime}}=0.89 ; \quad \frac{\beta_{c c c}}{\beta_{a c a}}=0.31
$$


TABLE III: Comparison of the bond additivity model and corrected bond additivity model values.

\begin{tabular}{ccccc}
\hline & \multicolumn{2}{c}{$\mathrm{CH}_{3} \mathrm{OH}$} & \multicolumn{2}{c}{$\mathrm{CH}_{3} \mathrm{CD}_{2} \mathrm{OH}$} \\
& BAM & Corrected & BAM & Corrected \\
\hline$R=\frac{\beta_{a a c}}{\beta_{c c c}}$ & 1.7 & 1.7 & 3.4 & 3.4 \\
$\mathrm{r}$ & 0.28 & 0.28 & 0.026 & 0.026 \\
$\frac{\beta_{c c c}}{\beta_{a c a}}$ & 1.1 & 3.8 & 0.30 & 0.31 \\
$\alpha_{c c}^{\prime} / \alpha_{a c}^{\prime}$ & 2.2 & 1.9 & 0.60 & 0.34 \\
$\mu_{c}^{\prime} / \mu_{a}^{\prime}$ & 0.48 & 1.9 & 0.48 & 0.89 \\
\hline
\end{tabular}

\section{Discussion}

Table III summarizes the values of $R, r, \beta_{c c c} / \beta_{a c a}$, $\alpha_{c c}^{\prime} / \alpha_{a c}^{\prime}, \mu_{c}^{\prime} / \mu_{a}^{\prime}$ in the uncorrected and corrected bond additivity model. It is easy to see that the biggest correction is the $\mu_{c}^{\prime} / \mu_{a}^{\prime}$ value for $\mathrm{CH}_{3} \mathrm{OH}$. According to the simple bond moment theory, $\mu_{c}^{\prime} / \mu_{a}^{\prime}=0.48$ for both $\mathrm{CH}_{3} \mathrm{OH}$ and $\mathrm{CH}_{3} \mathrm{CD}_{2} \mathrm{OH}$, i.e. the IR intensity for the $\mathrm{CH}_{3}$-as mode should be about $2(1 / 0.48)^{2}=8.7$ times of that for the $\mathrm{CH}_{3}$-ss mode. However, experimentally they are about 1.2 and 5 for $\mathrm{CH}_{3} \mathrm{OH}$ and $\mathrm{CH}_{3} \mathrm{CD}_{2} \mathrm{OH}$, respectively. Such big difference between these two molecules can be attributed to the fact that there is much stronger influence on the $\mathrm{CH}_{3}$ group stretching vibrations by the $\mathrm{O}-\mathrm{H}$ group in $\mathrm{CH}_{3} \mathrm{OH}$ than in $\mathrm{CH}_{3} \mathrm{CD}_{2} \mathrm{OH}$.

It is interesting to see that $r=0.026$ for $\mathrm{CH}_{3} \mathrm{CD}_{2} \mathrm{OH}$, while $r=0.28$ for $\mathrm{CH}_{3} \mathrm{OH}$. According to the definition of $r$ in Eq[15] $r=0$ means that each bond can be pictured as a perfect rod. Therefore, the value of $r$ may be used to correlate the coupling between the different bonds in the same group, and the perturbation of the bonds in the group by the connecting molecular groups. The $\mathrm{CH}_{3}$ group in $\mathrm{CH}_{3} \mathrm{OH}$ is likely to be much more strongly perturbed by the directly connecting $\mathrm{O}-\mathrm{H}$ group, than the $\mathrm{CH}_{3}$ group in $\mathrm{CH}_{3} \mathrm{CD}_{2} \mathrm{OH}$ by the directly connecting $C D_{2}$ and the indirectly connecting $\mathrm{O}-\mathrm{H}$ groups. The same effect as in $\mathrm{CH}_{3} \mathrm{OH}$ can also be expected for acetone and acetonitrile molecules, where the $\mathrm{CH}_{3}$ groups are directly connected to the $\mathrm{C}=\mathrm{O}$ and $-\mathrm{C} \equiv \mathrm{N}$ groups, respectively. Similarly, the longer chain alcohols should be similar to $\mathrm{CH}_{3} \mathrm{CD}_{2} \mathrm{OH}$. Therefore, it is not coincidental that the asymmetric $\mathrm{C}-\mathrm{H}$ stretching mode in the SFG-VS spectra from the vapor/acetone and vapor/acetonitrile interfaces has not been observed, ${ }^{23.62 .63 .64}$ while for the longer chain alcohols it has been experimentally observed $\stackrel{5.26 .48}{\underline{4}}$

If we put $I_{\text {sys }}^{\text {Raman }} / I_{\text {asym }}^{\text {Raman }}=5.0$ for $\mathrm{CH}_{3} \mathrm{OH}$ into Eq 17 we have $r=0.24$ for $0 \leq r \leq 1$; while if we put $I_{\text {sys }}^{\text {Raman }} / I_{\text {asym }}^{\text {Raman }}=0.54$ for $\mathrm{CH}_{3} \mathrm{CD}_{2} \mathrm{OH}$ into Eq17 there is no solution of $r$ for $0 \leq r \leq 1$ at all. This clearly shows that $\mathrm{Eq} 16$ and $\mathrm{Eq} 17$ do not generally give consistent $r$ values, and certainly they can not be held true at the same time.

Now, with the corrected $\beta_{c c c} / \beta_{\text {aca }}$ value for $\mathrm{CH}_{3} \mathrm{OH}$, the fact of missing $\mathrm{CH}_{3}$-as mode in SFG-VS from vapor $/ \mathrm{CH}_{3} \mathrm{OH}$ interface can be readily addressed. From the calculated intensities in Fig 2 with $\mathrm{Vis}=62^{\circ}$ and $\mathrm{IR}=53^{\circ}$ experimental configuration, $I_{C H_{3}-a s}^{s p s} / I_{C H_{3}-s s}^{s s p} \approx$ $1 / 2.5$ with the uncorrected bond additivity model value $\beta_{c c c} / \beta_{a c a}=1.1$, and with the known orientation angle $\theta \approx 0$ from previous studies 50.51 .53 Now, with the corrected value $\beta_{c c c} / \beta_{a c a}=3.8$, the $I_{\mathrm{CH}_{3}-a s}^{\text {sps }} / I_{\mathrm{CH}_{3}-s s}^{\text {ssp }}$ ratio should be $(3.8 / 1.1)^{2}=11.9$ times smaller, i.e., $I_{C H_{3}-a s}^{s p s} / I_{C H_{3}-s s}^{s s p} \approx 1 / 30$. It is easy to see that such a small $I_{\mathrm{CH}_{3}-a s}^{s p s}$ comparing to $I_{\mathrm{CH}_{3}-s s}^{s s p}$ is below the noise level and its spectral feature can not be observed in the SFG-VS spectra. Convincingly, using above approach, inspection of the IR and Raman spectra of liquid acetone and acetonitrile also indicated that the $\mathrm{CH}_{3}$-as vs. $\mathrm{CH}_{3}$-ss intensity ratio values are significantly smaller than the values calculated from the simple bond additivity model for their SFG-VS spectra from vapor/liquid interfaces 23.62 .63 .64

It is interesting to see that the correction for the $\beta_{c c c} / \beta_{a c a}$ value is very small for $\mathrm{CH}_{3} \mathrm{CD}_{2} \mathrm{OH}$, even though the corrections for $\alpha_{c c}^{\prime} / \alpha_{a c}^{\prime}$ and $\mu_{c}^{\prime} / \mu_{a}^{\prime}$ are not so small separately. These two corrections happened to cancel each other when used to calculate $\beta_{c c c} / \beta_{\text {aca }}$ with Eq 8 Using this ratio, the SFG-VS spectra of $\mathrm{CH}_{3} \mathrm{CD}_{2} \mathrm{OH}$ can be well interpreted just as with the uncorrected bond additivity model $\stackrel{10}{\underline{n}}$ However, it is to be noted that the success of the former is with firm empirical basis, and the success of the latter is nevertheless coincidental. We have performed measurements on the SFG-VS spectra in different incident angles for the vapor/ethanol interface, and analysis show that with the $\beta_{i^{\prime} j^{\prime} k^{\prime}}$ tensor ratios obtained here, all spectra as well as the spectra interference effects, can be quantitatively interpreted, and detailed molecular orientation analysis is also possible ${ }^{34}$

According to above discussion, the success of the corrected bond additivity model is evident. This success indicates that the failure of the bond additivity model discussed in Section IIB can be quantitatively corrected with the empirical approach as demonstrated here. However, we have to caution that this success is purely empirical, and may not be as general as it seems before further examinations being made. Even though it is undoubtedly an advancement from the simple bond additivity model, there are reasons this empirical approach may fail.

Because the correction for the bond additivity model used the Raman and IR spectra in the liquid phase, the success of this correction undoubtedly indicates that the $\mathrm{CH}_{3}$ group of these molecules under studying are not significantly different as in the liquid bulk phases or at the interfaces. This may only be true for the generally 'rigid' $\mathrm{C}-\mathrm{H}$ stretching vibrations which is usually not greatly perturbed and the assumption of local motion is generally valid. But this may not be true for the less 'rigid' molecular groups. Based on the successful treatment with the $\mathrm{CH}_{3}$ groups, we may assume that the same treatment on the similarly 'rigid' $\mathrm{NH}_{3}$ and $\mathrm{CH}_{2}$ groups is also going to work. If this approach fails to quantitatively interpret the SFG-VS spectra for a particular molecular group, one may try to consider the possibility that this group might have been very differently perturbed in the bulk phase from at the interface.

One clear advantage of this empirical approach is that 
the problem of the Fermi resonance and combinational mode couplings is automatically addressed through the empirical correction. As we have known, to assess the effects of such accidental resonances and couplings can be a very difficult problem in $a b$ initio calculations. Therefore, the empirical treatment discussed here may provide a way to quantitatively test the whole molecule approach ${ }^{14}$ For example, one direct may is to try to use the whole molecule approach to calculate the polarizability tensors of the $\mathrm{CH}_{3}$ group in the $\mathrm{CH}_{3} \mathrm{OH}$ and $\mathrm{CH}_{3} \mathrm{CD}_{2} \mathrm{OH}$, and compare the results directly with the IR, Raman and SFG-VS data. Provided with successful applications to a series of clearly studied molecular systems, one may expect that in the future $a b$ initio computation can be generally applied for quantitative interpretation of nonlinear spectroscopy.

\section{CONCLUSION}

In summary, we discussed a total empirical approach with symmetry analysis to quantitatively interpret observed SFG-VS spectra from molecular interfaces. In such an approach, the Raman and IR tensor ratios are directly calculated from Raman and IR spectral measurements using a certain correction procedure to the bond additivity model. This approach is tested with the SFGVS spectra from vapor $/ \mathrm{CH}_{3} \mathrm{OH}$ and vapor $/ \mathrm{CH}_{3} \mathrm{CD}_{2} \mathrm{OH}$ interfaces.

There have been few attempts to use Raman and IR spectra in the condensed phase to quantitatively interpret SFG-VS spectra from molecular interfaces in detail. It has been demonstrated that the bond additivity model has been successful in quantitative interpretation of SFGVS of the $\mathrm{CH}_{3}$ and $\mathrm{CH}_{2}$ as well as $\mathrm{H}_{2} \mathrm{O}$ groups for their ss modes, 10 but it has not been as successful with the as of these molecules or molecular groups. Through analysis of the problem we understood the empirical basis for the successes of the bond additivity model. So we proposed to treat the failures of the bond additivity model with a complete empirical approach. In this report we successfully demonstrated with two examples that the empirical corrections to the bond additivity model can successfully interpret what the simple bond additivity model failed. This development can become a new addition to the existing methodologies in quantitative SFG-VS studies. 10 We believe that this empirical approach can provide new understanding of the effectiveness and limitations of the bond additivity model, and this report also provided a practical roadmap for its application in SFG-VS studies of molecular interfaces.

Acknowledgment. HFW thanks supports by the Natural Science Foundation of China (NSFC No.20274055, No.20425309, No.20573117). ZFC thanks support by the Key Project of Ministry of Education of China (MOE No.204065).
APPENDIX: Terms and expressions for $\mathbf{C}_{2 v}$ group corrections

With the empirical approach derived and tested for the $\mathrm{C}_{3 v}$ groups, similar approach can be taken for the $\mathrm{C}_{2 v}$ groups. For them, bond additivity model has to be used to obtain necessary $\beta_{i^{\prime} j^{\prime} k^{\prime}}$ tensor ratios for quantitative calculation. $18,19.25 .26$

If the fixed coordinates system of the $\mathrm{AB}_{2}$ group with $\mathrm{C}_{2 v}$ symmetry is defined with $c$ axis as the symmetry axis, $a c$ plane as the A-B-A molecular plane, and $b$ is perpendicular to the molecular plane, then there are two A-B stretching vibrational mode with $A_{1}$ mode as the symmetric stretching (ss) vibrational mode, and $B_{1}$ mode the asymmetric (as) mode. $10,54,55$ The non-zero elements for the stretching vibrational modes are as the followings.

For the $A_{1}$ (symmetric) mode:

$$
\begin{array}{rlr}
\text { SFG-VS } & \beta_{a a c}=R_{a} \beta_{c c c} ; & \beta_{b b c}=R_{b} \beta_{c c c} \\
\text { Raman: } & \alpha_{a a}^{\prime}=R_{a} \alpha_{c c}^{\prime} ; & \alpha_{b b}^{\prime}=R_{b} \alpha_{c c}^{\prime} \\
\text { IR: } & \mu_{c}^{\prime}
\end{array}
$$

where $\mathrm{R}_{a}$ and $\mathrm{R}_{b}$ are the two $\beta_{i^{\prime} j^{\prime} k^{\prime}}$ elements ratios.

For the $B_{1}$ (asymmetric) mode:

$$
\begin{aligned}
\text { SFG-VS } & \beta_{a c a}=\beta_{c a a} \\
\text { Raman: } & \alpha_{a c}^{\prime}=\alpha_{c a}^{\prime} \\
\text { IR: } & \mu_{a}^{\prime}
\end{aligned}
$$

Therefore, $\mathrm{Eq} 9$ for IR intensity of the $C_{2 v}$ group becomes,

$$
\frac{I_{\text {sym }}^{I R}}{I_{\text {asym }}^{I R}}=\left(\frac{\mu_{c}^{\prime}}{\mu_{a}^{\prime}}\right)^{2}
$$

$\mathrm{Eq}$ 10.Eq13 are all generally valid for Raman measurement, 10,54,55 and Eq 14 alone cannot be used to determine both $\mathrm{R}_{a}$ and $\mathrm{R}_{b}$ values. Therefore, bond additivity model has to be employed.

For the single A-B bond treated with $C_{\infty v}$ symmetry, Eq 15 is generally assumed valid, and the definition of $a_{0}$ and $m_{0}$ of the single bond is the same as defines above. For the $\mathrm{C}_{2 v}$ group $\mathrm{AB}_{2}$ with the $\mathrm{A}-\mathrm{B}-\mathrm{A}$ angle as $\tau$, we have the following:

$$
\begin{aligned}
G_{A_{1}} & =\frac{1+\cos \tau}{M_{A}}+\frac{1}{M_{B}} \\
G_{B_{1}} & =\frac{1-\cos \tau}{M_{A}}+\frac{1}{M_{B}}
\end{aligned}
$$

in which $M_{A}$ and $M_{B}$ are the atomic masses of the atom $A$ and $B ; G_{A_{1}}$ and $G_{B_{1}}$ are the inverse reduced masses of the $A_{1}$ and $B_{1}$ normal modes, respectively, with the normal mode coordinates defined as,, 16.17 .58$

$$
\begin{aligned}
& Q_{A_{1}}=\left(\Delta r_{1}+\Delta r_{2}\right) /\left(2 G_{A_{1}}\right)^{1 / 2} \\
& Q_{B_{1}}=\left(\Delta r_{1}-\Delta r_{2}\right) /\left(2 G_{B_{1}}\right)^{1 / 2}
\end{aligned}
$$


Then the bond additivity model gives the following expressions of the non-zero $\alpha_{i^{\prime} j^{\prime}}^{\prime}$ and $\mu_{k^{\prime}}^{\prime}$ tensors.

For the $A_{1}$ (symmetric) mode:

$$
\begin{aligned}
& \alpha_{a a}^{\prime}=a_{0}\left[\sin ^{2}(\tau / 2)+r \cos ^{2}(\tau / 2)\right]\left(2 G_{A_{1}}\right)^{\frac{1}{2}} \\
& \alpha_{b b}^{\prime}=a_{0} r\left(2 G_{A_{1}}\right)^{\frac{1}{2}} \\
& \alpha_{c c}^{\prime}=a_{0}\left[r \sin ^{2}(\tau / 2)+\cos ^{2} \tau / 2\right]\left(2 G_{A_{1}}\right)^{\frac{1}{2}} \\
& \mu_{c}^{\prime}=m_{0} \cos (\tau / 2)\left(2 G_{A_{1}}\right)^{\frac{1}{2}}
\end{aligned}
$$

For the $B_{1}$ (asymmetric) mode:

$$
\begin{aligned}
& \alpha_{a c}^{\prime}=\alpha_{c a}^{\prime}=\frac{1}{2} a_{0}[(1-r) \sin \tau]\left(2 G_{B_{1}}\right)^{\frac{1}{2}} \\
& \mu_{a}^{\prime}=m_{0} \sin (\tau / 2)\left(2 G_{B_{1}}\right)^{\frac{1}{2}}
\end{aligned}
$$

Therefore, the bond additivity gives the following $\beta_{i^{\prime} j^{\prime} k^{\prime}}$ tensor ratios. 10

$$
\begin{aligned}
& R_{a}=\frac{\beta_{a a c}}{\beta_{c c c}}=\frac{1+r-(1-r) \cos \tau}{1+r+(1-r) \cos \tau} \\
& R_{b}=\frac{\beta_{b b c}}{\beta_{c c c}}=\frac{2 r}{1+r+(1-r) \cos \tau} \\
& R_{c / a}=\frac{\beta_{c c c}}{\beta_{a c a}}=\frac{\beta_{c c c}}{\beta_{c a a}} \\
& =\frac{[(1+r)+(1-r) \cos \tau] \cos (\tau / 2)}{(1-r) \sin \tau \sin (\tau / 2)} \frac{G_{A_{1}} \omega_{B_{1}}}{G_{B_{1}} \omega_{A_{1}}}
\end{aligned}
$$

with the $r$ values calculated from the experimentally obtained Raman depolarization ratio $\rho$ of the ss mode of the $\mathrm{AB}_{2}$ group using the following equation.

$$
\rho=\frac{3}{4+20(1+2 r)^{2} /\left[(1-r)^{2}\left(1+3 \cos ^{2} \tau\right)\right]}
$$

Following the example of the $\mathrm{C}_{3 v}$ group above, the procedure to make correction for the bond additivity model is as the following. First to use Eq 37 to obtain $r$ value. Then the $R_{a}$ and $R_{b}$ values can be obtained using this $r$ value. In order to calculate $R_{c / a}$, one need to use Eq 8 with the empirically corrected $\alpha_{c c}^{\prime} / \alpha_{a c}^{\prime}$ and $\mu_{c}^{\prime} / \mu_{a}^{\prime}$ values. The $\mu_{c}^{\prime} / \mu_{a}^{\prime}$ value can be obtained using Eq 34 Then the $\alpha_{c c}^{\prime} / \alpha_{a c}^{\prime}$ value can be obtained by put the $r$ value and the bond additivity model expressions for $\alpha_{i^{\prime} j^{\prime}}^{\prime}$ tensors above into Eq11 to derive a equation similar to Eq18

This correction procedure is certainly worth to be tested for interpretation of SFG-VS data of typical $\mathrm{C}_{2 v}$ molecules or molecular groups at interfaces. For example, at the air/water and organic/water interfaces, so far the asymmetric stretching mode of the $\mathrm{C}_{2 v}$ water molecule has not been clearly identified yet.19,21,22 Another example is that the intensity ratio between $\mathrm{CH}_{2}$-ss and $\mathrm{CH}_{2}$-as has been widely used to interpret $\mathrm{CH}_{2}$ group orientational changes at molecular and polymer interfaces 65
1 X. D. Zhu, H. Suhr, and Y. R. Shen, Phys. Rev. B 35, 3047 (1987).

2 Y. R. Shen, Nature 337, 519 (1989).

3 C. D. Bain, J. Chem. Soc. Faraday Trans. 91, 1281 (1995).

${ }^{4}$ K. B. Eisenthal, Chem. Rev. 96, 1343-1360 (1996).

5 P. B. Miranda, and Y. R. Shen, J. Phys. Chem. B 103, 3292 (1999).

6 M. J. Shultz, C. Schnitzer, D. Simonelli, and S. Baldelli, Int. Rev. Phys. Chem. 19, 123 (2000).

7 M. Buck, and M. Himmelhaus, J. Vac. Sci. Technol. A 19, 2717 (2001).

8 Z. Chen, Y. R. Shen, and G. A. Somorjai, Annu. Rev. Phys. Chem. 53, 437 (2002).

${ }^{9}$ G. L. Richmond, Chem. Rev. 102, 2693 (2002).

${ }^{10}$ H. F. Wang, W. Gan, R. Lu, Y. Rao, and B. H. Wu, Int. Rev. Phys. Chem. 24, 191 (2005).

11 P. Guyot-sionnest, J. H. Hunt, and Y. R. Shen, Phys. Rev. Letts. 59, 1597 (1987).

12 R. Superfine, J. Y. Huang, and Y. R. Shen, Phys. Rev. Letts. 66(8), 1066 (1991).

13 X. Zhuang, P. B. Miranda, D. Kim, and Y. R. Shen, Phys. Rev. B 59, 12632 (1999).

14 D. K. Hore, D. K. Beaman, D. H. Park, and G. L. Richmond, J. Phys. Chem. B 109, 16846 (2005).

15 Y. R. Shen, The Principles of Nonlinear Optics; John Wiley \& Sons: New York, 1984.

16 C. Hirose, N. Akamatsu, and K. Dom, J. Chem. Phys. 96, 997 (1992).

17 C. Hirose, H. Yamamoto, N. Akamatsu, and K. Domen, J. Phys. Chem. 97, 10064 (1993).

18 X. Wei, S-C. Hong, X. W. Zhuang, T. Goto, and Y. R. Shen, Phy. Rev. E 62, 5160 (2000).
19 Q. Du, R. Superfine, E. Freysz, and Y. R.Shen, Phys. Rev. Lett. 70, 2313 (1993).

20 A. Morita, J. T. Hynes, Chem. Phys. 258, 371 (2000).

21 W. Gan, D. Wu, Z. Zhang, Y. Guo, and H. F. Wang, Chin. J. Chem. Phys. in press.

${ }^{22}$ W. Gan, D. Wu, Z. Zhang, R. R. Feng, and H. F. Wang, J. Chem. Phys. in press.

23 Y. L. Yeh, C. Zhang, H. Held, A. M. Mebel, X. Wei, S. H. Lin, and Y. R. Shen, J. Chem. Phys. 114, 1837 (2001).

24 M. Oh-e, H. Yokoyama, and S. Baldelli, Appl. Phys. Letts. 84, 4965 (2004).

25 R. Lu, W. Gan, B. H. Wu, H. Chen, H. F. Wang, J. Phys. Chem. B 108, 7297 (2004).

26 R. Lu, W. Gan, B. H. Wu, Z. Zhang, Y. Guo, and H. F. Wang, J. Phys. Chem. B 109, 14118 (2005).

27 P. Fischer, and A. D. Buckingham, J. Opt. Soc. Am. B 15, 2951 (1998).

28 T. H. Ong, P. B. Davies, and C. D. Bain, Langmuir 9, 1836 (1993).

29 G. R. Bell, Z. X. Li, C. D. Bain, P. Fischer, and D. C. Duffy, J. Phys. Chem. B 102, 9461 (1998).

30 J. L. McHale, Molecular Spectroscopy (Prentice Hall, Inc., 1999). pp280.

31 D. Zhang, J. Gutow, and K. B. Eisenthal, J. Phys. Chem. 98, 13729-13734 (1994).

32 E. Tyrode, C. M. Johnson, S. Baldelli, C. Leygraf, and M. W. Rutland, J. Phys. Chem. B 109, 329-341 (2005)

33 Y. Rao, M. Comstock, and K. B. Eisenthal, J. Phys. Chem. B 110, jp055340r (2006). In press.

${ }^{34}$ W. Gan, B. H. Wu, Z. Zhang, Y. Guo, and H. F. Wang, to be submitted.

${ }^{35}$ K. M. Gough, J. Chem. Phys. 91, 2424 (1989). 
${ }^{36}$ L. Dixit, P. Kumar, R. B. Gupta, and P. L. Gupta, Appl. Spectro. Rev. 18, 373 (1982).

37 M. V. Wolkenshtein, M. A. Eliashevich, and B. I. Stepnov, Kolebaniyia Molecule(Vibrations of Molecules), Vol.1, GITIL, Moscow, 1949.

${ }^{38}$ L. A. Gribov, Intensity Theory for Infrared Spectra of Polyatonic Molecules, Consultants Bureau, New York,1964.

39 S. Abbate, M. Gussoni, and G. Zeribi, Indian J. Pure Appl. Phys. 16, 199 (1978)

40 T. Yoshino, and H. J. Bernstein, J. Mol. Spec. 2, 213 (1958).

41 D. Steele, Quat. Rev. 18, 21 (1964).

42 I. C. Hisatsune, J. Chem. Phys. 23, 487 (1955).

43 D. F. Eggers, I. C. Hisatsune, L. V. Alten, J. Phys. Chem. 59, 1124 (1955).

44 D. F. Hornig, D. C. Mckean, J. Phys. Chem. 59, 1133 (1955).

45 D. C. Harris, and M. D. Bertolucci, Symmetry and Spectroscopy: An Introduction to Vibrational and Electronic Spectroscopy, Dover Publications: New York, 1990.

46 K. Wolfrum, H. Graener, and A. Laubereau, Chem. Phys. Lett. 213, 41-46 (1993).

47 J. Y. Huang, M. H. Wu, Phys. Rev. E 50, 3737-3746 (1994).

48 C. D. Stanners, Q. Du, R. P. Chin, P. Cremer, G. A. Somorjai, and Y. R. Shen, Chem. Phys. Letts. 232, 407-413 (1995)

49 G. Ma, and H. C. Allen, J. Phys. Chem. B 107, 6343-6349 (2003).

50 (a) R. Lu, W. Gan, and H. F. Wang, Chin. Sci. Bull. 48, 2183-2187 (2003); (b) R. Lu, W. Gan, and H. F. Wang, Chin. Sci. Bull. 49, 899 (2004).

${ }^{51}$ H. Chen, W. Gan, R. Lu, Y. Guo, and H. F. Wang, J.
Phys. Chem. B 109, 8064-8075 (2005).

52 J. Sung, K. Park, and D. Kim, J. Phys. Chem. B 109 , 18507-18514 (2005).

53 W. Gan, B. H. Wu, H. Chen, Y. Guo, and H. F. Wang, Chem. Phys. Lett. 406, 467-473 (2005).

54 D. A. Long, The Raman effect: A Unified Treatment of the Theory of Raman Scattering by Molecules (John Wiley and Sons, Chichester, 2002).

55 L. A. Nafie, and W. L. Peticolas, J. Chem. Phys. 57, 3145 (1972).

56 M. J. Colles, and J. E. Griffiths, J. Chem. Phys. 56, 3384 (1972).

57 D.A.Long, Raman Spectroscopy, McGraw-Hill, New York 1977.

58 Bao-hua Wu, Ph.D. Thesis, Institute of Chemistry, Chinese Academy of Sciences, No. 200218003200664, Beijing, July 2005.

59 Private communication with Professor Shi-lin Liu at Department of Chemical Physics, University of Science and technology of China.

60 J. E. Bertie, and S. L. Zhang, J. Mol. Structure 413-414, 333 (1997).

61 J-P. Perchard, and M-L. Josien, J. Chim. Phys. 65, 1856 (1968).

62 H. Chen, W. Gan, B. H. Wu, D. Wu, Y. Guo, and H. F. Wang, J. Phys. Chem. B 109, 8053-8063 (2005).

${ }^{63}$ H. Chen, W. Gan, B. H. Wu, D. Wu, Z. Zhang, and H. F. Wang, Chem. Phys. Lett. 408, 284-289 (2005).

64 J. Kim, K. C. Chou, and G. A. Somorjai, J. Phys. Chem. B. 107, 1592-1596 (2003).

65 A. Opdahl, R. A. Phillips, and Gabor A. Somorjai, J. Phys. Chem. B 106, 5212-5220 (2002). 